\title{
Clostridium difficile Colitis in the Hospital Setting: A Potentially Explosive Problem
}

\author{
Charles W. Stratton, MD
}

Pseudomembranous enterocolitis was first described long before the antibiotic era by Coats in 1883. ${ }^{1}$ Initially, this clinical syndrome was recognized in postoperative hospitalized patients, and because of its distinctive pseudomembrane, it was termed postoperative diphtheritic enteritis in 1893 by Finney. ${ }^{2}$ Shortly after the introduction of antibiotics, there were several cases of a presumably new clinical syndrome of severe enteritis with extensive pseudomembrane formation in patients receiving antibiotics. ${ }^{3}$ This was called antibioticassociated pseudomembranous colitis. In the first reported cases, necropsy revealed enteritis with pseudomembranes under which pure cultures of Staphylococcus aureus usually could be isolated. It was reasoned that antibiotic use allowed the overgrowth of $S$ aureus, which then produced a toxic enterocolitis. 4 This supposition was strengthened by the observations of staphylococcal-induced enterocolitis in an animal model. ${ }^{5}$

However, doubt was cast upon this hypothesis by noting that the syndrome had first been described in the pre-antibiotic era and that identical enterocolitis had been found in patients whose stool cultures did not grow staphylococci. ${ }^{6}$ The role of $\mathrm{S}$ aureus in enterocolitis is still controversial; cases may occur, particularly with "toxic shock" strains, but these appear to be exceedingly rare.

The etiology of most cases of pseudomembranous enterocolitis began to be unraveled when Larson and colleagues reported a nondialyzable heat-labile cytotoxin in the stools of five of six patients with antibiotic-associated pseudomembranous colitis. ${ }^{7}$ Using a hamster model described by Small, ${ }^{8}$ the

\footnotetext{
From the Department of Pathology, Clinical Microbiology, Vanderbilt University, Nashville, Tennessee.

Address reprint requests to Charles W. Stratton, MD, Director, Clinical Microbiology, Department of Pathology, C-3213, Medical Center North, Vanderbilt University, Nashville, TN 37232.

Stratton CW. Clostridium difficile colitis in the hospital setting: a potentially explosive problem. Infect Control Hosp Epidemiol. 1990;11:281-282.
}

same heat-labile cytotoxic material found in patients with clindamycin-associated pseudomembranous colitis could be found in hamsters receiving clindamycin. 9,10 The cytotoxic effect could be neutralized by antiserum against Clostridium difficile and Clostridium sordellii, but not by specific antisera against other Clostridium species. Finally, the same cytotoxic activity was demonstrated in broth culture filtrates of $C$ difficile strains isolated from patients with antibiotic-associated colitis. Thus, most cases of antibiotic-associated pseudomembranous colitis are now associated with cytotoxigenic $\mathrm{C}$ difficile. ${ }^{11}$

$C$ difficile was first described in 1935 by Hall and O'Toole, ${ }^{12}$ who found this anaerobic bacillus colonizing newborns. This microogranism was initially named Bacillus difficilis because of the difficulties encountered in growing this anaerobe in cultures. Although only a colonizer of newborns, this organism was noted to be pathogenic for guinea pigs and rabbits. Snyder, in 1937, noted that certain strains of $B$ difficilis produced a thermolabile toxin that was lethal for guinea pigs and that the guinea pigs could be protected with specific antiserum. ${ }^{13}$

Pseudomembranous enterocolitis has been associated with a number of antibiotics besides clindamycin. ${ }^{14}$ In addition, case clusters ${ }^{15}$ suggest $C$ difficile spores ${ }^{16}$ may transmit this illness in the nosocomial setting. ${ }^{17}$ In this issue of Infection Control and Hospital Epidemiology, Brown and colleagues report a 100 -fold increase in the hospital-wide attack rate for $C$ difficile colitis over a five-year span. Risk factors included the use of antibiotics, older age, hospitalization in an intensive care unit and nonsurgical gastrointestinal manipulation. The association with antibiotic use implicated both clindamycin and third-generation cephalosporins. The former agent is well-known for its association with antibiotic-induced colitis; the third-generation cephalosporins, on the other hand, are not, and would not be suspected at first glance because of their minimal activity against anaerobic organisms. 
It should be realized that the third-generation cephalosporins do have activity against many anaerobes other than those in the Bacteroides fragilis group. Ceftriaxone is largely excreted via the biliary tract and could easily alter the gastrointestinal flora. Ceftizoxime has been reported to have activity similar to that of cefoxitin against members of the $B$ fragilis group. ${ }^{18}$ These two agents, plus ceftazidime, were implicated along with clindamycin in the study by Brown, et al. This study did not investigate the potential role of nosocomial transmission in the increase in colitis, but others ${ }^{17}$ have demonstrated the importance of nosocomial acquisition of $C$ difficile colitis.

The study does point out the explosive potential for $C$ difficile colitis in the hospital setting, Recognition of this potential and appropriate screening should be included in infection control programs at other hospitals. This increase in colitis was controlled by restricting clindamycin use and encouraging metronidazole use. These control measures, plus the recommendation of early isolation of infected patients, are prudent and should be considered by infection control committees in other hospitals. In this DRG era, the increased morbidity and costs associated with a 100 -fold increase in C difficile colitis is something no hospital can afford.

\section{REFERENCES}

1. Coats J. A Manual of Pathology. Philadelphia, Pa: Henry C. Lea's Sons; 1883.

2. Finney JMT Gastroenterostomy for cicatrizing ulcer of the pylorus.
Bull Johns Hopkins Hosp. 1893;4:53-54.

3. Reiner L, Schlesinger MJ, Miller GM. Pseudo-membranous colitis following aureomycin and chloramphenicol. Arch Pathol.1952;54:3967.

4. Dearing WH, Baggenstoss AH, Weed LA. Studies on the relationship of Staphylococcus aureus to pseudomembranous enteritis and to postantibiotic enteritis. Gastroenterology. 1960;38:441-451.

5. Bennett IL, Wood JS, Yardley JH. Staphylococcal psuedomembraneous enterocolitis in chinchillas: a clinico-pathologic study. Trans Assoc Am Physicians. 1956;69:116-121.

6. Hardaway RM, McKay DG. Pseudomembranous enterocolitis: are antibiotics wholly responsible? Arch Surg. 1959;78:457-466.

7. Larson HE, Parry JV, Price AB, et al. Undescribed toxin in pseudomembranous colitis. Br Med J. 1977;1:1246-1248.

8. Small JD. Fatal enterocolitis in hamsters given lincomycin hydrochloride. Laboratory Animal Care. 1968;18:411-420.

9. Bartlett JG, Chang TW, Gurwith M, Gorbach SL, Onderdonk AB. Antibiotic-associated pseudomembranous colitis due to toxinproducing clostridia. N Eng J Med. 1978;298:531-534.

10. George RH. Symonds JM, Dimock F, et al. Identification of Clostridium difficile as a cause of pseudomembranous colitis. $\mathrm{Br}$ Med J. 1978;1:695.

11. Bartlett JG, Chang TW, Taylor NS, Onderdonk AB. Colitis induced by Clostridium difficile. Reu Infect Dis. 1979;1:370-378.

12. Hall IC, O'Toole E. Intestinal flora in new-bom infants with a description of a new pathogenic anaerobe, Bacillus difficilis. Am J Dis Child. 1935;49:390-402.

13. Snyder ML. Further studies on Bacillus difficilis. J Infect Dis. 1937;60:223-231

14. Silva J. Fekety R, Werk C, et al. Inciting and etiologic agents of colitis. Rev Infect Dis. 1984;6(suppl):214-221.

15. Kabins SA. Outbreak of clindamycin-associated colitis. Ann Intern Med. 1975;83:830-831.

16. Kim KH, Fekety R, Batts $\mathrm{DH}$, et al. Isolation of Clostridium difficile from the environment and contacts of patients with antibiotic associated colitis. J Infect Dis. 1981;143:42-50.

17. McFarland LV, Mulligan M, Kwok RYY, Stamm WE. Nosocomial acquisition of Clostridium difficile infection. N Engl $J$ Med. $1989 ; 320: 204-210$

18. O'Keefe JP, Venezio FR. Divincenzo CA. Shatzer KL. Activity of newer $\beta$-lactam agents against clinical isolates of Bacteroides fragilis and other Bacteroides species. Antimicrob Agents Chemother. 1987;31:2002-2004. 